\title{
A prospective study of statin use and mortality among 67,385 blacks and whites in the Southeastern United States
}

This article was published in the following Dove Press journal:

Clinical Epidemiology

18 December 2013

Number of times this article has been viewed

\author{
Loren Lipworth' \\ Sergio Fazio ${ }^{1,2}$ \\ Edmond K Kabagambe \\ Heather M Munro ${ }^{3}$ \\ Victor C Nwazue' \\ Robert E Tarone ${ }^{3}$ \\ Joseph K McLaughlin ${ }^{3}$ \\ William J Blot ${ }^{1,3}$ \\ Uchechukwu KA \\ Sampson ${ }^{1,2,4}$ \\ 'Department of Medicine, \\ Vanderbilt University Medical \\ Center, ${ }^{2}$ Department of Pathology, \\ Microbiology and Immunology, \\ Vanderbilt University Medical Center, \\ Nashville, TN, USA; ${ }^{3}$ International \\ Epidemiology Institute, Rockville, \\ MD, USA; ${ }^{4}$ Department of Radiology \\ and Radiological Sciences, Vanderbilt \\ University Medical Center, Nashville, \\ TN, USA
}

Correspondence: Loren Lipworth Vanderbilt University Medical Center, Division of Epidemiology, 2525 West End Avenue, Suite 600, Nashville, TN, USA 37203

Tel +l 6153430639

Email loren.lipworth@vanderbilt.edu
Purpose: The primary objective of this study is to examine the race-specific associations between statin use and overall mortality, as well as cardiovascular and cancer mortality, among blacks and whites in the Southeastern United States (US). Little is known about these associations in blacks.

Patients and methods: The Southern Community Cohort Study is an ongoing, prospective cohort study, which enrolled from 2002 through 2009 nearly 86,000 participants aged 40-79 years. We used Cox regression models to estimate race-specific hazard ratios (HRs) and 95\% confidence intervals (CI) for overall and cause-specific mortality associated with statin use based on self-reported hypercholesterolemia and treatment at cohort entry. Mean age at cohort entry was 51.4 years in blacks $(n=48,825)$ and 53.5 years in whites $(n=18,560)$. Sixty-one percent of participants were women. Whites were more likely to have self-reported hypercholesterolemia (40\% versus $27 \%, P<0.001$ ), and to report being treated with either statins ( $52 \%$ versus $47 \%$, $P<0.001)$ or combination lipid therapy (9\% versus $4 \%, P<0.001)$ compared with blacks, regardless of sex. During follow-up (median: 5.6 years) 5,199 participants died. Compared with untreated hypercholesterolemic individuals, statin use was associated with reduced all-cause mortality (adjusted HR [aHR] 0.86; 95\% CI 0.77-0.95) and cardiovascular disease mortality overall (aHR 0.75; 95\% CI 0.64-0.89) and among whites (aHR 0.67; 95\% CI 0.50-0.90), blacks (aHR, 0.80; 95\% CI 0.65-0.98), men (aHR 0.70; 95\% CI 0.55-0.90), and women (aHR 0.79; 95\% CI 0.63-0.99) ( $P>0.05$ for race and sex interaction). Statin use was not associated with cancer mortality overall or in subgroup analyses.

Conclusion: Regardless of race or sex, self-reported statin use was linked to reduced all-cause and cardiovascular disease mortality. However, factors contributing to the modestly lower statin use and markedly lower prevalence of self-reported hypercholesterolemia among blacks remain to be determined.

Keywords: statins, disparities, hypercholesteromia, undertreatment, underdiagnosis, mortality

\section{Introduction}

After decades of clinical trials, 3-hydroxy-methylglutaryl coenzyme A reductase inhibitors (statins) have emerged as key agents for reducing cardiovascular events and mortality via the modulation of inflammation and serum lipoprotein cholesterol levels. ${ }^{1-3}$ However, data are limited on the use of statins across ethnic groups, and it is not known whether racial differences exist in the relationship between statin use and cardiovascular mortality, particularly among blacks for whom rates of cardiovascular disease (CVD) mortality are still the highest in the United States (US). Moreover, the benefit of statins on all-cause mortality has not been conclusively demonstrated for women. ${ }^{4,5}$ Finally, despite early animal studies indicating an association between 
statins and increased risk of tumors, ${ }^{6}$ substantial experimental data suggest that statins may in fact have antiproliferative and proapoptotic effects. ${ }^{7,8}$ Epidemiologic studies of the association between statin use and cancer have generated inconsistent results, ${ }^{9,10}$ and few studies have included black participants.

The Southern Community Cohort Study (SCCS) is a large, prospective cohort study examining health disparities among adults, over two-thirds black, residing in the Southeastern US where rates of CVD are among the highest in the nation. ${ }^{11}$ The similar income and education levels of both black and white participants in the SCCS allow contemporaneous socioeconomic differences that sometimes confound racial comparisons to be minimized. Thus, we have characterized the use of statins among black and white participants in the SCCS, and we have examined race-specific associations between baseline statin use and all-cause, cardiovascular, and cancer mortality. Our a priori analysis objectives also included examination of the association between statin use and mortality separately among men and women, as well as by diabetic status and age $(<65$ or $\geq 65$ years) at cohort entry.

\section{Methods}

The SCCS is an ongoing, prospective cohort study, which enrolled from 2002 through 2009 nearly 86,000 adult participants, age 40-79 years, residing in 12 states in the Southeastern US. The study design and methods have been described in detail previously. ${ }^{11}$ Approximately $86 \%$ of participants were enrolled through participating community health centers $(\mathrm{CHC})$, institutions which provide primary health and preventive services in medically underserved areas and thus serve generally low-income populations, ${ }^{12}$ and the remaining 14\% were enrolled through mail-based general population sampling. The present report focuses on 67,385 black and white $\mathrm{CHC}$ participants, which ensured similarities in socioeconomic characteristics and access to health care at cohort entry regardless of race. The restriction to black and white participants was because the sample sizes for other racial groups were too small for stable statistical analysis.

Upon entry into the SCCS, participants were administered a baseline, computer-assisted personal interview at the CHC (available at http://www.southerncommunitystudy. org), which ascertained information about demographic, socioeconomic, and anthropometric characteristics, personal and family medical history, tobacco and alcohol use, medication use, and other factors. Participants were asked whether a doctor had ever told them they had high cholesterol.
Those who responded affirmatively were asked additional follow-up questions regarding the use (and names) of all prescription medications for treatment of high cholesterol. Participants were classified into four groups based on their responses to these questions: no high cholesterol at baseline, high cholesterol with no lipid-lowering drug therapy, high cholesterol on statin therapy, high cholesterol on other drug therapy. SCCS participants provided written informed consent, and the Institutional Review Boards of Vanderbilt University Medical Center and Meharry Medical College approved all study protocols.

Vital status and cause of death were ascertained by linkage of the cohort with the Social Security Administration and the National Death Index (NDI), respectively. For analyses of all-cause mortality and cause-specific mortality, followup extended through December 31, 2010 (the latest date for which cause of death information was available from NDI) or date of death, whichever occurred first. For those whose vital status was reported as unknown by the Social Security Administration in 2010, follow-up was censored at the last SCCS contact with the participant if prior to December 31, 2010. The following cause-specific categories of mortality were examined: CVD (underlying causes classified as International Classification of Diseases, Tenth Revision [ICD-10], codes I00-I99, which include ischemic heart diseases, hypertensive diseases, cerebrovascular diseases, and other forms of heart disease); cancer (ICD-10 codes C00-C97); and all other non-external causes excluding CVD, cancer, and all ICD-10 codes beginning with $\mathrm{S}, \mathrm{T}, \mathrm{V}, \mathrm{W}, \mathrm{X}$, and $\mathrm{Y}$ (such as injury, poisoning, or accident).

\section{Statistical analysis}

Crude frequency distributions of categorical variables were compared using chi-square tests, while $t$-tests or analysis of variance were used for continuous variables. Cox proportional hazards models, using age as the time scale, were used to estimate hazard ratios (HRs) and corresponding 95\% confidence intervals $(\mathrm{CI})$ for the relation between statin use and cause-specific and all-cause mortality overall and by age, race, sex, and diabetic status. Data satisfied the proportional hazards assumption, and the following covariates were selected a priori based on subject matter knowledge: year of SCCS enrollment (2002-2003, 2004-2005, 2006-2007, 2008-2009); marital status (married, single, divorced, or widowed); highest level of education completed ( $<$ high school, high school/vocational school, some college/ junior college, completed college, or graduate school); total household income in the previous year $(<\$ 15,000$; 
$\$ 15,000-\$ 24,999 ; \$ 25,000-\$ 49,999 ; \geq \$ 50,000)$; health insurance status (none, any private insurance, Medicaid only, Medicare only, other); body mass index (BMI) using participants' self-reported weight and height at baseline (BMI $<18.5$ defines underweight, 18.5-24.9 normal weight, 25-29.9 overweight, 30-39.9 obese, $\geq 40$ morbidly obese); cigarette smoking (never, former, current $<10$ cigarettes per day [cpd], 10-19 cpd, and $\geq 20 \mathrm{cpd}$ ); alcohol consumption (none, moderate [one drink/day for women, two drinks/ day for men], heavy [ $>$ one drink/day for women, $>$ two drinks/day for men]); and history of hypertension, myocardial infarction (MI)/coronary bypass surgery (CABG), diabetes, and stroke (all yes/no). Differences between crude and adjusted HR estimates were minimal and we present only the adjusted estimates. A two-tailed $P$-value $<0.05$ was accepted as the threshold for statistical significance. Statistical analyses were conducted using SAS software, version 9.3 (SAS Institute Inc., Cary, NC, USA).

Our a priori analysis plan called for examination of the association between statin use and mortality separately by race and sex, as well as by diabetic status and age $(<65$ or $\geq 65$ years) at cohort entry. Likelihood ratio tests were conducted comparing the main effects models with and without interaction terms between statin use and race, sex, diabetes status, or age.

\section{Results}

Among the 67,385 SCCS participants included in this analysis, $72 \%(\mathrm{n}=48,825)$ were black and $61 \%(\mathrm{n}=40,777)$ were women. Mean age at start of follow-up was 51.4 and 53.5 years for blacks and whites, respectively. Overall, $88 \%$ of participants had no history of MI/CABG or stroke at baseline, while $56 \%$ and $21 \%$ reported having been diagnosed with hypertension or diabetes, respectively, prior to cohort enrollment.

At the baseline interview, 20,722 (31\%) participants reported a diagnosis of high cholesterol (Table 1). The prevalence of high cholesterol was significantly higher among whites than blacks $(40 \%$ versus $27 \% ; P<0.001)$ (Table 2), and this racial difference was evident for both men (34\% for whites, $21 \%$ for blacks) and women ( $43 \%$ for whites, $32 \%$ for blacks), and for all age and income groups. Among participants with high cholesterol, 10,045 (48\%) reported being treated with a statin and 9,783 (47\%) were untreated, while only 894 (4\%) reported using other lipid lowering drugs, including 228 users of fibrates and 172 users of ezetimibe. Mean age at diagnosis of high cholesterol was younger among untreated individuals ( 45.7 years), compared with those treated with statins (49.8 years) or other drugs (48.8 years), and this pattern was observed for all race and sex groups.

The frequency of statin use was significantly higher $(P<0.001)$ among whites than blacks with self-reported high cholesterol ( $52 \%$ versus $47 \%$ ), among whites than blacks overall (21\% versus $13 \%)$, and among women than men with high cholesterol (49\% versus $47 \%$ ) and overall (17\% versus $11 \%$ ). Similarly, the frequency of combination therapy (statin plus other lipid-lowering drug) was higher in whites than blacks ( $9 \%$ versus $4 \%, P<0.001)$. Approximately $90 \%$ of statin users used lipophilic statins, with the majority using atorvastatin.

Distributions of the four study groups according to baseline characteristics are presented in Table 1. Statin use increased during the study period, from a prevalence of $12 \%$ in 2002-2003 to a prevalence of $18 \%$ in 2008-2009. Statin use also increased with increasing age of the participant. Statin use was not consistently associated with education level and income, but those who smoked cigarettes or drank alcohol were less likely to be statin users than non-smokers or non-drinkers. BMI was strongly associated with use of statins; compared with normal weight (BMI 18.5-24.9) individuals, the prevalence of statin use among those who were extremely obese (BMI $\geq 40$ ) was more than three times higher. Participants with high cholesterol and with any of the evaluated comorbidities (hypertension, $\mathrm{MI} / \mathrm{CABG}$, diabetes, or stroke) were significantly more likely to be treated with statins. For all of the examined variables, patterns of association with statin use were virtually identical among blacks and whites (Table 2), although the higher use among women than men was more pronounced among blacks (15\% versus $9 \%)$ than whites ( $22 \%$ versus $18 \%)$.

Median duration of follow-up was 5.6 years, accounting for 352,389 person-years overall. When examined for subgroups, median follow-up (years) was 5.9 for blacks, 4.7 for whites, 5.6 for women, 5.5 for men, 5.1 for statin users, and 5.7 for non-users of statins. During the follow-up, a total of 5,199 deaths were identified in the cohort, 819 of them among statin users. CVD was the cause of death for 1,643 deaths, 316 among statin users.

\section{All-cause, CVD, and cancer mortality}

Compared with those with untreated high cholesterol, statin users experienced significantly lower all-cause mortality and CVD mortality, with HRs of 0.86 (95\% CI 0.77-0.95) and 0.75 (95\% CI 0.64-0.89), respectively (Table 3). Decreased all-cause mortality associated with statin use 
Table I Self-reported high cholesterol and statin or other lipid-lowering medication use according to baseline characteristics of 67,385 SCCS participants, 2002-2009

\begin{tabular}{|c|c|c|c|c|}
\hline \multirow[t]{2}{*}{ Characteristic } & \multicolumn{3}{|l|}{$\begin{array}{l}\text { High cholesterol } \\
n=20,722(31 \%)\end{array}$} & \multirow{2}{*}{$\begin{array}{l}\text { No high cholesterol } \\
n=46,663(69 \%) \\
n(\%)\end{array}$} \\
\hline & $\begin{array}{l}\text { Statins }^{b} \\
n=10,045 \text { (I5\%) } \\
n(\%)\end{array}$ & $\begin{array}{l}\text { Other drugs } \\
n=894(1 \%) \\
n(\%)\end{array}$ & $\begin{array}{l}\text { Untreated } \\
n=9,783 \text { (15\%) } \\
n(\%)\end{array}$ & \\
\hline \multicolumn{5}{|l|}{ Age at interview (years) } \\
\hline $40-49$ & $2,448(7)$ & $246(1)$ & $4,109(13)$ & $25,938(79)$ \\
\hline $50-59$ & $3,873(17)$ & $337(2)$ & $3,602(16)$ & $14,5 \mid 6(65)$ \\
\hline $60-69$ & $2,791(30)$ & $223(2)$ & $\mathrm{I}, 576(17)$ & $4,7 \mid 3(5 \mid)$ \\
\hline 70-79 & $933(31)$ & $88(3)$ & $496(16)$ & $\mathrm{I}, 496(50)$ \\
\hline \multicolumn{5}{|l|}{ Year of SCCS enrollment } \\
\hline $2002-2003$ & $2,589(12)$ & $210(1)$ & $2,990(14)$ & $15,5 \mid 2(73)$ \\
\hline 2004-2005 & $2,958(14)$ & $257(1)$ & $3,005(14)$ & $14,702(70)$ \\
\hline $2006-2007$ & $2,787(18)$ & $270(2)$ & $2,435(15)$ & $10,360(65)$ \\
\hline 2008-2009 & $1,711(18)$ & $157(2)$ & I,353 (I5) & $6,089(65)$ \\
\hline \multicolumn{5}{|l|}{ Sex } \\
\hline Female & $7,042(17)$ & $574(I)$ & $6,653(16)$ & $26,508(65)$ \\
\hline Male & $3,003(11)$ & $320(1)$ & $3,130(12)$ & $20,155(76)$ \\
\hline \multicolumn{5}{|l|}{ Race } \\
\hline Black & $6,220(13)$ & $487(1)$ & $6,623(14)$ & $35,495(73)$ \\
\hline White & $3,825(21)$ & $407(2)$ & $3,160(17)$ & $11,168(60)$ \\
\hline \multicolumn{5}{|l|}{ Marital status } \\
\hline Married & $3,797(18)$ & $348(2)$ & $3,301(16)$ & $13,764(65)$ \\
\hline Single & $\mathrm{I}, 40 \mathrm{I}(9)$ & $123(1)$ & $1,853(12)$ & $|2,73|(79)$ \\
\hline Divorced & $3,237(14)$ & $294(1)$ & $3,554(15)$ & $16,357(70)$ \\
\hline Widowed & $1,609(24)$ & $129(2)$ & $1,073(16)$ & $3,80 \mathrm{I}(57)$ \\
\hline \multicolumn{5}{|l|}{ Education } \\
\hline$<$ high school & $3,492(16)$ & $27 \mid(1)$ & $3,044(14)$ & $14,434(68)$ \\
\hline High/vocational school & $3,901(14)$ & $343(1)$ & $3,730(14)$ & $19,382(71)$ \\
\hline Some college & $\mathrm{I}, 744(\mathrm{I} 4)$ & $187(1)$ & $2,006(16)$ & $8,779(69)$ \\
\hline$\geq$ college & $906(15)$ & $93(2)$ & $1,002(17)$ & $4,060(67)$ \\
\hline \multicolumn{5}{|c|}{ Annual household income (\$) } \\
\hline$<15,000$ & $5,999(15)$ & $503(1)$ & $5,684(14)$ & $28,358(70)$ \\
\hline $15,000-24,999$ & $2,191(15)$ & $169(1)$ & $2,063(14)$ & $10,337(70)$ \\
\hline $25,000-49,999$ & I,I89 (I5) & $147(2)$ & $\mid, 311(16)$ & $5,387(67)$ \\
\hline$\geq 50,000$ & $562(17)$ & $66(2)$ & $601(18)$ & $2,126(63)$ \\
\hline \multicolumn{5}{|l|}{ Health insurance } \\
\hline Any private & $2,250(16)$ & $205(1)$ & $2,289(16)$ & $9,472(67)$ \\
\hline Medicare only & $\mathrm{I}, 644(26)$ & $128(2)$ & $998(16)$ & $3,507(56)$ \\
\hline Medicaid only & $1,466(16)$ & $125(I)$ & I,277 (I4) & $6,576(70)$ \\
\hline Other & $1,700(23)$ & $192(3)$ & $1,189(16)$ & $4,459(59)$ \\
\hline No health insurance & $2,932(10)$ & $243(1)$ & $3,958(13)$ & $22,407(76)$ \\
\hline \multicolumn{5}{|l|}{ BMI $\left(\mathrm{kg} / \mathrm{m}^{2}\right)$} \\
\hline Underweight $(<18.5)$ & $36(4)$ & $2(0)$ & $80(9)$ & $762(87)$ \\
\hline Normal (18.5-24.9) & $\mathrm{I}, \mathrm{I} 88(7)$ & $114(1)$ & $\mathrm{I}, 760(\mathrm{II})$ & $|3,5| \mid(82)$ \\
\hline Overweight (25-29.9) & $2,666(14)$ & $254(1)$ & $2,899(15)$ & $|3,55|(70)$ \\
\hline Obese (30-39.9) & $4,448(20)$ & $380(2)$ & $3,80 I(17)$ & $13,903(62)$ \\
\hline Extremely obese (40+) & $1,618(22)$ & $138(2)$ & $1,162(16)$ & $4,467(60)$ \\
\hline \multicolumn{5}{|l|}{ Smoking } \\
\hline Never & $4,018(17)$ & $351(2)$ & $3,658(16)$ & $15,343(66)$ \\
\hline Former & $3,096(22)$ & $270(2)$ & $2,435(18)$ & $8,089(58)$ \\
\hline Current $<10$ & $848(9)$ & $74(1)$ & $\mathrm{I}, 075(\mathrm{II})$ & $7,726(79)$ \\
\hline Current 10-19 & $923(9)$ & $69(1)$ & $\mathrm{I}, 179(12)$ & $7,715(78)$ \\
\hline Current 20+ & $\mathrm{I}, \mathrm{I} 48$ (II) & $130(1)$ & I,428 (I4) & $7,7 \mid 3(74)$ \\
\hline \multicolumn{5}{|l|}{ Alcohol drinking ${ }^{a}$} \\
\hline Heavy & $709(6)$ & $72(\mathrm{I})$ & $\mathrm{I}, 347$ (I I) & 10,302 (83) \\
\hline Moderate & $2,892(13)$ & $264(1)$ & $3,377(15)$ & $16,539(72)$ \\
\hline None & $6,347(20)$ & $55 I(2)$ & $4,951(16)$ & $19,444(62)$ \\
\hline
\end{tabular}


Table I (Continued)

\begin{tabular}{|c|c|c|c|c|}
\hline \multirow[t]{2}{*}{ Characteristic } & \multicolumn{3}{|l|}{$\begin{array}{l}\text { High cholesterol } \\
n=20,722(31 \%)\end{array}$} & \multirow{2}{*}{$\begin{array}{l}\text { No high cholesterol } \\
n=46,663(69 \%) \\
n(\%)\end{array}$} \\
\hline & $\begin{array}{l}\text { Statins }^{b} \\
n=10,045 \text { ( I 5\%) } \\
n(\%)\end{array}$ & $\begin{array}{l}\text { Other drugs } \\
n=894(1 \%) \\
n(\%)\end{array}$ & $\begin{array}{l}\text { Untreated } \\
n=9,783(15 \%) \\
n(\%)\end{array}$ & \\
\hline \multicolumn{5}{|l|}{ Hypertension } \\
\hline No & I,688 (6) & $163(1)$ & 3,266 (II) & $24,453(83)$ \\
\hline Yes & $8,356(22)$ & $731(2)$ & $6,512(17)$ & $22,192(59)$ \\
\hline \multicolumn{5}{|l|}{$\mathrm{MI} / \mathrm{CABG}$} \\
\hline No & 8,174 (13) & $733(1)$ & $9,028(14)$ & 44,99I (7I) \\
\hline Yes & $1,859(42)$ & $161(4)$ & $744(17)$ & $\mathrm{I}, 643(37)$ \\
\hline \multicolumn{5}{|l|}{ Diabetes } \\
\hline No & $5,416(10)$ & $496(1)$ & $7,384(14)$ & $39,884(75)$ \\
\hline Yes & $4,624(33)$ & $398(3)$ & $2,392(17)$ & $6,762(48)$ \\
\hline \multicolumn{5}{|l|}{ Stroke } \\
\hline No & $8,703(14)$ & $780(1)$ & $8,957(14)$ & 44,424 (7I) \\
\hline Yes & $|, 33|(30)$ & $113(3)$ & $803(18)$ & $2,210(50)$ \\
\hline
\end{tabular}

Notes: ${ }^{M}$ Moderate (one drink/day for women, two drinks/day for men) and heavy (>one drink/day for women, $>$ two drinks/day for men); ${ }^{\text {b }}$-values for all comparisons were $<0.0001$.

Abbreviations: BMI, body mass index; CABG, coronary artery bypass surgery; MI, myocardial infarction; SCCS, Southern Community Cohort Study.

was more pronounced among whites (HR $0.82 ; 95 \% \mathrm{CI}$ 0.69-0.98) than blacks (HR 0.89; 95\% CI 0.79-1.02); the same was true for CVD mortality, with HRs of 0.67 (95\% CI $0.50-0.90)$ among whites and 0.80 (95\% CI $0.65-0.98)$ among blacks. The race-by-statin use interaction was not statistically significant for either all-cause $(P=0.19)$ or CVD mortality $(P=0.22)$. Statin use was more strongly inversely associated with all-cause and CVD mortality among men ( $\mathrm{HR}=0.82,95 \% \mathrm{CI} 0.70-0.96$, and $\mathrm{HR}=0.70,95 \% \mathrm{CI}$ $0.55-0.90$, respectively) than women (HR $=0.89,95 \%$ CI $0.77-1.02$, and HR $=0.79,95 \%$ CI $0.63-0.99$, respectively), although the interaction between sex and statin use was not statistically significant for all-cause $(P=0.49)$ or CVD mortality $(P=0.39)$.

The beneficial effects of statins on both all-cause and CVD mortality were evident among diabetics (HR $0.81 ; 95 \%$ CI $0.70-0.94$ and HR $0.71 ; 95 \%$ CI $0.56-0.90$, respectively) and non-diabetics (HR 0.89; 95\% CI 0.77-1.03 and HR 0.79; 95\% CI 0.63-1.00, respectively). Statin use was inversely associated with total mortality regardless of age, with HRs of 0.85 (95\% CI $0.76-0.96)$ and 0.85 (95\% CI $0.68-1.06)$ among those below and above age 65 years, respectively. For CVD mortality, the corresponding HRs were 0.75 (95\% CI $0.62-0.90)$ and 0.83 (95\% CI 0.58-1.20) ( $P>0.05$ for all interactions) (data not shown).

There was no association between statin use and cancer deaths overall (HR $=0.90 ; 95 \%$ CI $0.72-1.12)$ and by race, sex, or baseline diabetes status (Table 3). Additional adjustment of the models for history of cancer at baseline had virtually no effect on the HRs for cancer mortality.
Participants with untreated high cholesterol at baseline had slightly lower HRs for all-cause mortality compared to those without high cholesterol, regardless of race or sex, which was accounted for by increases in non-CVD mortality among the group without high cholesterol (HR for cancer $=1.19,95 \%$ CI $0.99-1.42$; HR for other external causes $=1.34 ; 95 \%$ CI 1.16-1.55; Table 3). CVD mortality rates were similar among those with untreated high cholesterol and those with normal cholesterol.

\section{Discussion}

In this SCCS population of predominantly low-income blacks and whites in the Southeastern US, including some of the largest numbers of women and blacks studied in a single study to date, we observed a significant $14 \%$ reduction in HR of all-cause mortality among statin-treated versus untreated patients with hypercholesterolemia. The absence of an association between statin use and death from cancer or other nonvascular causes suggests that the beneficial effect on all-cause mortality is driven mainly by the strong reduction (25\%) in the HR of CVD mortality observed among statin users. Similar decreases in risk of total and cardiovascular death among users of statins have been consistently reported, ${ }^{5,13-17}$ including in the primary prevention setting, although an atorvastatin trial failed to show benefits on all-cause mortality. ${ }^{16}$

Our study population provides for the first time not only a description of statin use patterns among a large number of blacks, but also prospectively derived risk estimates of the total and cardiovascular mortality benefits of 


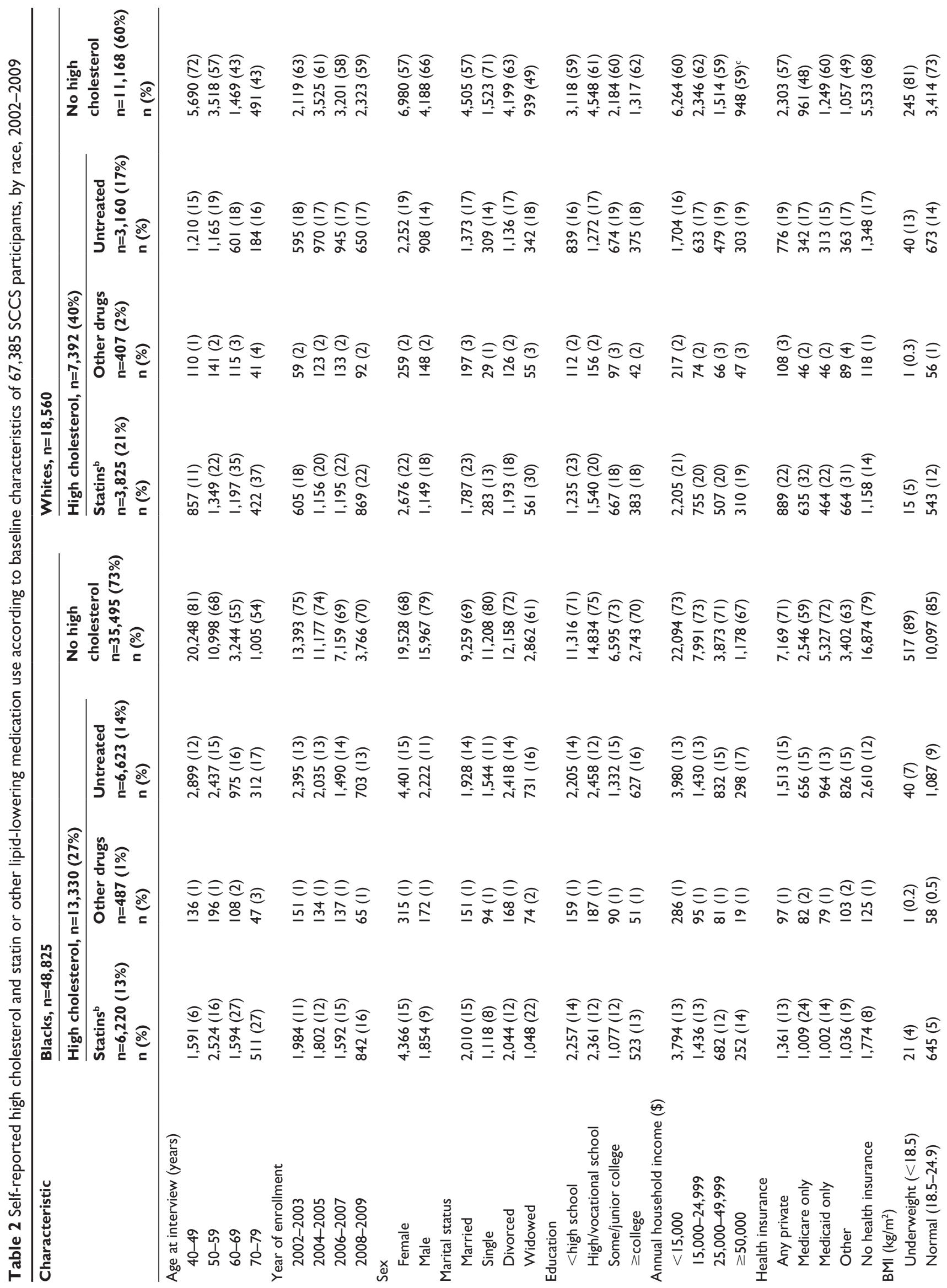




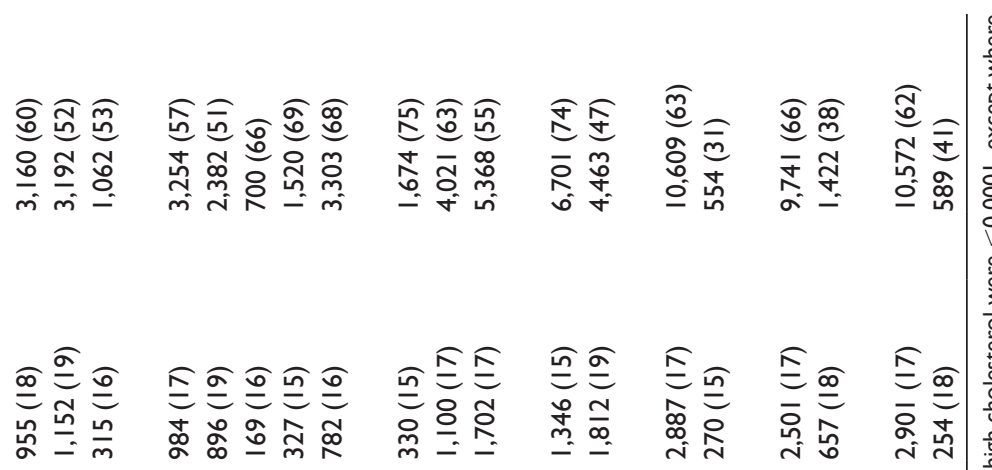

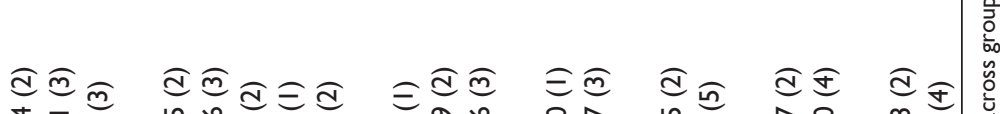

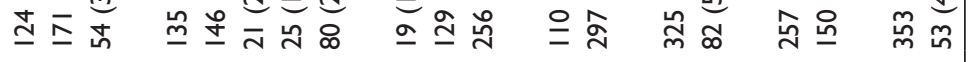

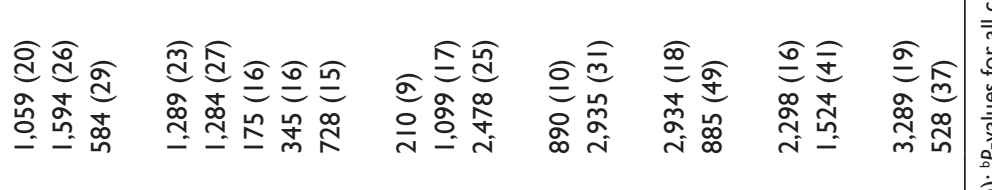

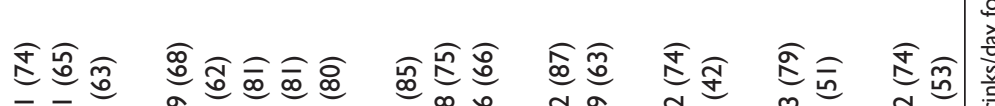

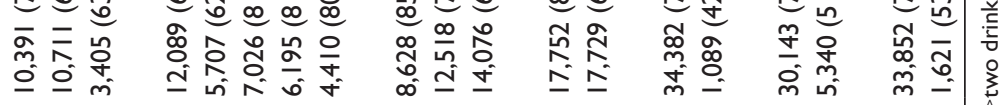

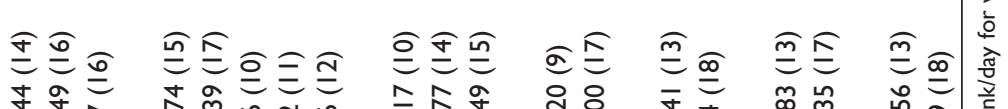

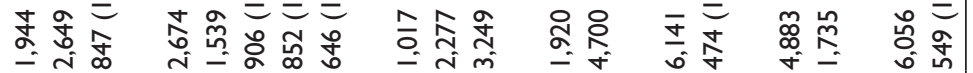

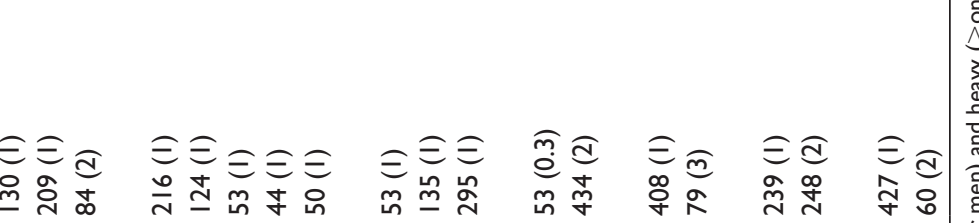

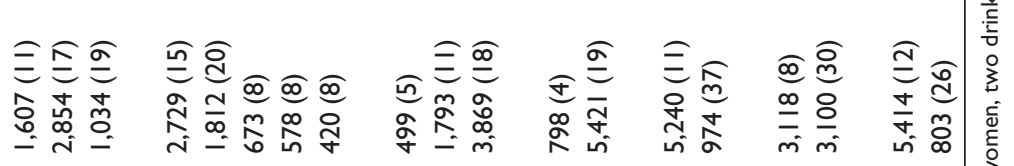

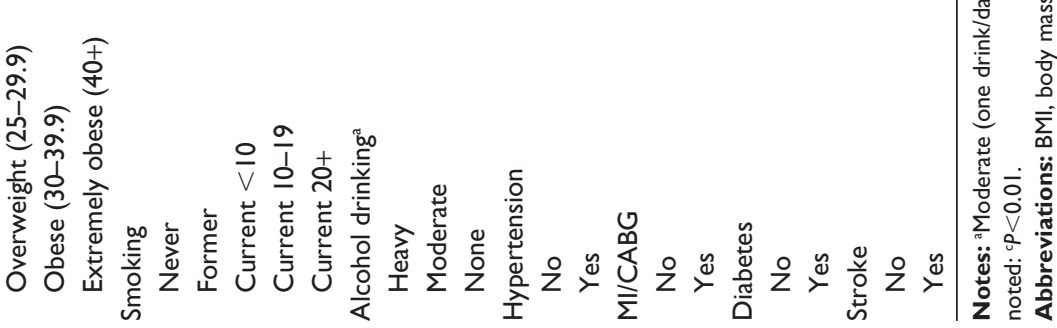


Table 3 Hazard ratios (HRs) and 95\% confidence intervals (Cls) for all-cause and cause-specific mortality in relation to high cholesterol and use of statins at cohort entry

\begin{tabular}{|c|c|c|c|c|c|c|c|c|}
\hline & \multicolumn{4}{|l|}{ Overall } & \multicolumn{4}{|l|}{ Blacks } \\
\hline & Deaths & PY & $\mathbf{H R}^{\mathbf{a}}$ & $95 \% \mathrm{Cl}$ & Deaths & PY & $\mathbf{H R}^{\mathbf{a}}$ & $95 \% \mathrm{Cl}$ \\
\hline \multicolumn{9}{|l|}{ All deaths } \\
\hline High cholesterol, statins & 819 & 49,329 & 0.86 & $0.77-0.95$ & 505 & 32,365 & 0.89 & $0.79-1.02$ \\
\hline High cholesterol, other drugs & 86 & 4,274 & 0.98 & $0.78-1.22$ & 53 & 2,468 & 1.10 & $0.82-1.46$ \\
\hline No high cholesterol & 3,600 & 248,251 & 1.15 & $1.06-1.25$ & 2,685 & 196,359 & 1.16 & $1.05-1.28$ \\
\hline High cholesterol, untreated & 694 & 50,534 & Ref & & 464 & 35,976 & Ref & \\
\hline \multicolumn{9}{|c|}{ Cardiovascular deaths (ICD-10 100-199) } \\
\hline High cholesterol, statins & 316 & & 0.75 & $0.64-0.89$ & 204 & & 0.80 & $0.65-0.98$ \\
\hline High cholesterol, other drugs & 28 & & 0.73 & $0.50-1.08$ & 14 & & 0.64 & $0.37-1.09$ \\
\hline No high cholesterol & $\mathrm{I}, 026$ & & 0.94 & $0.82-1.07$ & 803 & & 0.97 & $0.82-1.14$ \\
\hline High cholesterol, untreated & 273 & & Ref & & 190 & & Ref & \\
\hline \multicolumn{9}{|l|}{ Cancer deaths (ICD-10 C00-C97) } \\
\hline High cholesterol, statins & 167 & & 0.90 & $0.72-1.12$ & 99 & & 0.92 & $0.69-1.21$ \\
\hline High cholesterol, other drugs & 14 & & 0.87 & $0.50-|.5|$ & 8 & & 0.95 & $0.46-1.95$ \\
\hline No high cholesterol & 859 & & 1.19 & $0.99-1.42$ & 663 & & 1.21 & $0.97-1.49$ \\
\hline High cholesterol, untreated & 152 & & Ref & & 102 & & Ref & \\
\hline \multicolumn{9}{|l|}{ All other non-external causes ${ }^{\mathrm{b}}$} \\
\hline High cholesterol, statins & 289 & & 0.94 & $0.79-1.12$ & 173 & & 0.93 & $0.75-1.17$ \\
\hline High cholesterol, other drugs & 37 & & 1.29 & $0.91-1.83$ & 27 & & 1.69 & I. $12-2.55$ \\
\hline No high cholesterol & I,303 & & 1.34 & $1.16-1.55$ & 950 & & 1.29 & $1.08-1.53$ \\
\hline High cholesterol, untreated & 218 & & Ref & & 148 & & Ref & \\
\hline
\end{tabular}

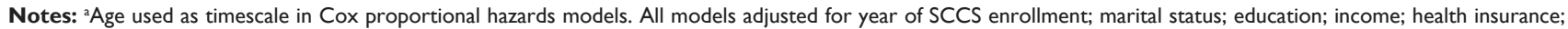
BMI; cigarette smoking; alcohol consumption; history of hypertension, MI/CABG, diabetes, and stroke; and for race and sex where appropriate; bexcludes deaths due to CVD, cancer, and external cause (ICD-I0 codes beginning with $\mathrm{S}, \mathrm{T}, \mathrm{V}, \mathrm{W}, \mathrm{X}$, and $\mathrm{Y}$ ).

Abbreviations: BMI, body mass index; CABG, coronary artery bypass surgery; CVD, cardiovascular disease; ICD-I0, International Classification of Diseases, Tenth Revision; MI, myocardial infarction; PY, person-years; Ref, reference category; SCCS, Southern Community Cohort Study.

statin use among blacks. We did not expect to observe a marked difference in the prevalence of self-reported hypercholesterolemia between white (40\%) and black (27\%) participants in our study. A difference of this magnitude would be difficult to explain even if black populations showed lower mean low-density lipoprotein (LDL) cholesterol levels compared to whites, which is not the case; a recent report of trends in lipids and lipoproteins in US adults over a 22 -year period, ${ }^{18}$ as well as the distribution of plasma LDL cholesterol levels among black and white participants in the Atherosclerosis Risk in Communities Study, ${ }^{19}$ do not show significant differences between blacks and whites. Consequently, it is reasonable to entertain the suspicion of underdiagnosis or decreased awareness of this condition in blacks. This is concerning, particularly in the context of the similarities in socioeconomic characteristics and access to care among members of the SCCS cohort.

Among those with high cholesterol, the frequency of use of statin or combination lipid-lowering therapy was modestly but significantly higher among whites; this racial difference was evident for both men and women, suggesting undertreatment among blacks even when more aggressive management is needed. Racial differences in utilization of antihypertensive medications in this same cohort were not observed (data not shown), suggesting that systematic error or general underreporting by blacks are unlikely to explain these disconcerting results. Of note, the pattern of dyslipidemia and use of lipid-lowering medications observed in this cohort mirrors trends in US adults by age, sex, and race. ${ }^{18}$ These findings, in conjunction with the inverse association between statin use and lifestyle factors such as smoking and alcohol consumption, have important implications for future research and clinical practice related to the aggressive treatment of high cholesterol in populations with high rates of CVD events and related risk factors.

Statins decrease LDL cholesterol levels by about the same amount in women as in men. A recent meta-analysis of 18 randomized trials, which included over 40,000 women, reported similar reductions in cardiovascular event rates and all-cause mortality among men and women who used statins, ${ }^{5}$ although a number of trials have failed to show substantial or statistically significant all-cause mortality benefits in women. ${ }^{4}$ In our large study population of over 40,000 women, we observed moderately stronger effect sizes among men, but substantial 21\%-30\% reductions in HRs of CVD mortality and $11 \%-18 \%$ reductions in HRs of all-cause 


\begin{tabular}{|c|c|c|c|c|c|c|c|c|c|c|c|}
\hline \multicolumn{4}{|l|}{ Whites } & \multicolumn{4}{|l|}{ Men } & \multicolumn{4}{|c|}{ Women } \\
\hline Deaths & PY & $\mathbf{H R}^{\mathbf{a}}$ & $95 \% \mathrm{Cl}$ & Deaths & PY & $\mathbf{H R}^{\mathbf{a}}$ & $95 \% \mathrm{Cl}$ & Deaths & PY & $\mathbf{H R}^{\mathbf{a}}$ & $95 \% \mathrm{Cl}$ \\
\hline 314 & 16,965 & 0.82 & $0.69-0.98$ & 351 & & 0.82 & $0.70-0.96$ & 468 & & 0.89 & $0.77-1.02$ \\
\hline 33 & 1,806 & 0.84 & $0.58-1.22$ & 46 & & 0.99 & $0.73-1.36$ & 40 & & 0.92 & $0.67-1.28$ \\
\hline 915 & 51,892 & 1.15 & $0.99-1.33$ & 2,116 & & 1.12 & $0.99-1.26$ & $\mathrm{I}, 484$ & & 1.17 & $1.04-1.32$ \\
\hline 230 & 14,559 & Ref & & 327 & & Ref & & 367 & & Ref & \\
\hline 112 & & 0.67 & $0.50-0.90$ & 143 & & 0.70 & $0.55-0.90$ & 173 & & 0.79 & $0.63-0.99$ \\
\hline 14 & & 0.85 & $0.48-1.50$ & 14 & & 0.65 & $0.37-1.13$ & 14 & & 0.80 & $0.46-1.38$ \\
\hline 223 & & 0.87 & $0.67-1.12$ & 618 & & 0.92 & $0.76-1.11$ & 408 & & 0.94 & $0.77-1.14$ \\
\hline 83 & & Ref & & 134 & & Ref & & 139 & & Ref & \\
\hline 68 & & 0.84 & $0.58-1.23$ & 66 & & 0.95 & $0.66-1.37$ & 101 & & 0.89 & $0.67-1.18$ \\
\hline 6 & & 0.75 & $0.32-1.77$ & 7 & & 1.04 & $0.47-2.28$ & 7 & & 0.77 & $0.35-1.65$ \\
\hline 196 & & 1.15 & $0.84-1.58$ & 475 & & 1.24 & $0.94-1.64$ & 384 & & 1.14 & $0.91-1.43$ \\
\hline 50 & & Ref & & 59 & & Ref & & 93 & & Ref & \\
\hline 116 & & 0.99 & $0.73-1.34$ & 118 & & 0.88 & $0.67-1.15$ & $17 \mid$ & & 0.99 & $0.78-1.27$ \\
\hline 10 & & 0.84 & $0.43-1.63$ & 19 & & 1.28 & $0.78-2.09$ & 18 & & 1.25 & $0.76-2.07$ \\
\hline 353 & & 1.45 & $1.12-1.88$ & 748 & & 1.23 & $|.00-| .5 \mid$ & 555 & & 1.43 & I.16-1.75 \\
\hline 70 & & Ref & & 105 & & Ref & & 113 & & Ref & \\
\hline
\end{tabular}

mortality among both men and women. Interestingly, in the meta-analysis, ${ }^{5}$ the all-cause mortality benefit among women was more pronounced among patients with low cardiovascular risk profiles. The majority of participants in our study overall had no reported history of $\mathrm{MI} / \mathrm{CABG}$ or stroke at baseline, and obesity, despite its high prevalence in this cohort, has been shown to be associated with elevated all-cause and CVD mortality only among white women in the SCCS. ${ }^{20}$ Thus, the mortality benefit observed among women in our cohort appears to support the finding of the meta-analysis, although $19 \%$ of those on statins had a prior $\mathrm{MI} / \mathrm{CABG}$ so may be treated for secondary rather than primary prevention.

The use of statins as a strategy for CVD risk reduction in the elderly and among diabetics has important public health implications. In line with results of a recent meta-analysis, ${ }^{21}$ we observed similar beneficial effects of statins on all-cause and CVD mortality among those with and without diabetes at baseline, and among those older or younger than 65 . Evaluation of increased risk of developing incident diabetes among statin users is of increasing concern ${ }^{22,23}$ and warrants further investigation with longer follow-up in the SCCS population.
Cancer mortality was not significantly associated with statin use overall or in any examined subgroup in our study. Despite a few reports of modest protective effects of statins on cancer, numerous reviews and meta-analyses of more than 38 observational studies and clinical trials have similarly concluded that statin use is not associated with cancer incidence or mortality overall or at specific sites. ${ }^{9,10}$ A recent large prospective Danish study (162,067 cancer deaths) showed an inverse association between statin use and cancer mortality among those using statins at the time of cancer diagnosis. It is possible that we may have missed detecting an association due to the low number of cancer events during the relatively short follow-up in our cohort and small effect size of statins on cancer. ${ }^{24}$ In any case, it is reassuring that statin use for primary prevention of CVD does not appear to be associated with increased cancer risk.

We did observe modestly increased risks of death from cancer and non-CVD causes, but similar CVD mortality, among those with normal cholesterol compared with those with untreated high cholesterol. This finding is consistent with previous epidemiologic studies of serum total or LDL cholesterol, particularly among the elderly. ${ }^{25-28}$ However, rather than causal associations, these are likely to reflect 
unhealthy lifestyle choices of the normal cholesterol group, who are more likely to be current smokers and heavy alcohol drinkers, or complex physician decision-making leading perhaps to underprescribing of statins to hypercholesterolemic patients with fewer or less severe risk factors and lower LDL levels. ${ }^{29,30}$

Among the limitations of our study is the fact that we were unable to assess duration of statin therapy, or dose or adherence. Moreover, the study relied on self-report of high cholesterol and statin use, which could result in possible misclassification effects of unknown magnitude and direction, and we had no information on when the individual initiated statin therapy. As prevalent or long-term users of statins represent a select population of individuals who tolerate therapy and have survived earlier events, inclusion of these patients may bias the results of observational studies. An additional limitation is reliance on ICD-10 cause of death codes from the National Death Index, which may not correctly classify all causes of death. Residual confounding by comorbidities or other variables for which information was not obtained on the baseline questionnaire, known and unknown biases associated with the prescription of statins, ${ }^{31,32}$ and increased health consciousness and healthier lifestyle among statin users, ${ }^{33,34}$ which are difficult to measure in an observational study, cannot be ruled out and could explain in part the observed beneficial effects of statins. Duration of follow-up was relatively short in our study (median 5.6 years), and further follow-up of the cohort for examination of the studied associations is warranted. Furthermore, the equivalent HR for CVD mortality among those with untreated high cholesterol and those with normal cholesterol raise the possibility that benefits of statins on CVD may derive not only from lowering of cholesterol but also from anti-inflammatory or other attributes of the medications.

The major strengths of our study include the large sample size and the comparison of black and white populations of similar socioeconomic status and comparable health care access, thus allowing examination of mortality effects of statins by race while minimizing confounding effects related to socioeconomic status. The study cohort was enrolled in a single region of 12 states in the Southeastern US, with little likelihood of additional influences from subregional factors. Our choice of the referent group (hypercholesterolemic not on lipid-lowering drugs instead of normolipidemic participants) may have helped to reduce the potential bias due to differential awareness of existence of hypercholesterolemia. In addition, the collection of extensive baseline information for the entire SCCS cohort, and the systematic follow up for all-cause and cause-specific mortality, enabled robust, precise estimation of multivariate HRs associated with use of statins in the largest cohort of blacks studied to date.

\section{Conclusion}

We have observed associations suggesting generally similar and strong benefits of statin use on overall and CVD mortality among both blacks and whites in the Southeastern US. The low prevalence of self-reported hypercholesterolemia in blacks raises the possibility of underdiagnosis of this strongest of cardiovascular risk factors, and our findings suggest modest underutilization of statins among blacks. Whether these results are generalizable to black and white populations in regions of the US other than the Southeast, in which rates of CVD are particularly high, warrants further investigation.

\section{Acknowledgments}

The Southern Community Cohort Study (SCCS) is funded by grant R01 CA092447 from the National Cancer Institute (NCI), including American Recovery and Reinvestment Act Funding (3R01 CA029447-08 S1). Dr Sampson's effort was supported in part by Harold Amos Medical Faculty Award of the Robert Wood Johnson Foundation. William J Blot (Principal Investigator of SCCS) and Heather Munro (staff statistician) had full access to all of the data in the study and take responsibility for the integrity of the data and the accuracy of the data analysis.

\section{Disclosure}

The authors report no conflicts of interest in this work.

\section{References}

1. Grundy SM, Cleeman JI, Merz CN, et al; National Heart, Lung, and Blood Institute; American College of Cardiology Foundation; American Heart Association. Implications of recent clinical trials for the National Cholesterol Education Program Adult Treatment Panel III guidelines. Circulation. 2004;110(2):227-239.

2. Palaniswamy C, Selvaraj DR, Selvaraj T, Sukhija R. Mechanisms underlying pleiotropic effects of statins. Am J Ther. 2010;17(1):75-78.

3. Smith SC Jr, Allen J, Blair SN, et al; AHA/ACC; National Heart, Lung, and Blood Institute. AHA/ACC guidelines for secondary prevention for patients with coronary and other atherosclerotic vascular disease: 2006 update: endorsed by the National Heart, Lung, and Blood Institute. Circulation. 2006;113(19):2363-2372.

4. Gutierrez J, Ramirez G, Rundek T, Sacco RL. Statin therapy in the prevention of recurrent cardiovascular events: a sex-based meta-analysis. Arch Intern Med. 2012;172(12):909-919.

5. Kostis WJ, Cheng JQ, Dobrzynski JM, Cabrera J, Kostis JB. Metaanalysis of statin effects in women versus men. $J$ Am Coll Cardiol. 2012;59(6):572-582.

6. Newman TB, Hulley SB. Carcinogenicity of lipid-lowering drugs. JAMA. 1996;275(1):55-60. 
7. Chan KK, Oza AM, Siu LL. The statins as anticancer agents. Clin Cancer Res. 2003;9(1):10-19.

8. Friis S, Olsen JH. Statin use and cancer risk: an epidemiologic review. Cancer Invest. 2006;24(4):413-424.

9. Browning DR, Martin RM. Statins and risk of cancer: a systematic review and metaanalysis. Int J Cancer. 2007;120(4):833-843.

10. Dale KM, Coleman CI, Henyan NN, Kluger J, White CM. Statins and cancer risk: a meta-analysis. JAMA. 2006;295(1):74-80.

11. Signorello LB, Hargreaves MK, Blot WJ. The Southern Community Cohort Study: investigating health disparities. $J$ Health Care Poor Underserved. 2010;21(Suppl 1):26-37.

12. Hargreaves MK, Arnold CW, Blot WJ. Community health centers: Their role in the treatment of minorities and in health disparities research. In: Satcher D, Pamies R, editors. Multicultural Medicine and Health Disparities. New York: McGraw-Hill; 2006:485-494.

13. Cholesterol Treatment Trialists' (CTT) Collaborators, Mihaylova B, Emberson J, et al. The effects of lowering LDL cholesterol with statin therapy in people at low risk of vascular disease: meta-analysis of individual data from 27 randomised trials. Lancet. 2012;380(9841): 581-590.

14. Gardette V, Bongard V, Dallongeville J, et al. Ten-year all-cause mortality in presumably healthy subjects on lipid-lowering drugs (from the Prospective Epidemiological Study of Myocardial Infarction [PRIME] prospective cohort). Am J Cardiol. 2009;103(3):381-386.

15. Kearney PM, Blackwell L, Collins R, et al; Cholesterol Treatment Trialists' (CTT) Collaborators. Efficacy of cholesterol-lowering therapy in 18,686 people with diabetes in 14 randomised trials of statins: a metaanalysis. Lancet. 2008;371(9607):117-125.

16. LaRosa JC, Grundy SM, Waters DD, et al; Treating to New Targets (TNT) Investigators. Intensive lipid lowering with atorvastatin in patients with stable coronary disease. $N$ Engl J Med. 2005;352(14):1425-1435.

17. Ridker PM, Pradhan A, MacFadyen JG, Libby P, Glynn RJ. Cardiovascular benefits and diabetes risks of statin therapy in primary prevention: an analysis from the JUPITER trial. Lancet. 2012;380(9841):565-571.

18. Carroll MD, Kit BK, Lacher DA, Shero ST, Mussolino ME. Trends in lipids and lipoproteins in US adults, 1988-2010. JAMA. 2012;308(15): 1545-1554.

19. Cohen JC, Boerwinkle E, Mosley TH Jr, Hobbs HH. Sequence variations in PCSK9, low LDL, and protection against coronary heart disease. N Engl J Med. 2006;354(12):1264-1272.

20. Cohen SS, Signorello LB, Cope EL, et al. Obesity and all-cause mortality among black adults and white adults. Am J Epidemiol. 2012;176(5):431-442
21. Brugts JJ, Yetgin T, Hoeks SE, et al. The benefits of statins in people without established cardiovascular disease but with cardiovascular risk factors: meta-analysis of randomised controlled trials. BMJ. 2009;338: b2376.

22. Sampson UK, Linton MF, Fazio S. Are statins diabetogenic? Curr Opin Cardiol. 2011;26(4):342-347.

23. Rajpathak SN, Kumbhani DJ, Crandall J, Barzilai N, Alderman M, Ridker PM. Statin therapy and risk of developing type 2 diabetes: a meta-analysis. Diabetes Care. 2009;32(10):1924-1929.

24. Nielsen SF, Nordestgaard BG, Bojesen SE. Statin use and reduced cancer-related mortality. $N$ Engl J Med. 2012;367(19):1792-1802.

25. Alsheikh-Ali AA, Trikalinos TA, Kent DM, Karas RH. Statins, lowdensity lipoprotein cholesterol, and risk of cancer. J Am Coll Cardiol. 2008;52(14):1141-1147.

26. Kronmal RA, Cain KC, Ye Z, Omenn GS. Total serum cholesterol levels and mortality risk as a function of age. A report based on the Framingham data. Arch Intern Med. 1993;153(9):1065-1073.

27. Krumholz HM, Seeman TE, Merrill SS, et al. Lack of association between cholesterol and coronary heart disease mortality and morbidity and all-cause mortality in persons older than 70 years. JAMA. 1994;272(17):1335-1340.

28. Newson RS, Felix JF, Heeringa J, Hofman A, Witteman JC, Tiemeier H Association between serum cholesterol and noncardiovascular mortality in older age. J Am Geriatr Soc. 2011;59(10):1779-1785.

29. Verma A, Visintainer P, Elarabi M, Wartak S, Rothberg MB. Overtreatment and undertreatment of hyperlipidemia in the outpatient setting. South Med J. 2012;105(7):329-333

30. Yang CC, Jick SS, Testa MA. Who receives lipid-lowering drugs: the effects of comorbidities and patient characteristics on treatment initiation. Br J Clin Pharmacol. 2003;55(3):288-298.

31. Glynn RJ, Knight EL, Levin R, Avorn J. Paradoxical relations of drug treatment with mortality in older persons. Epidemiology. 2001;12(6): 682-689.

32. Glynn RJ, Schneeweiss S, Wang PS, Levin R, Avorn J. Selective prescribing led to overestimation of the benefits of lipid-lowering drugs. J Clin Epidemiol. 2006;59(8):819-828.

33. Brookhart MA, Patrick AR, Dormuth C, et al. Adherence to lipid-lowering therapy and the use of preventive health services: an investigation of the healthy user effect. Am J Epidemiol. 2007;166(3):348-354.

34. Patrick AR, Shrank WH, Glynn RJ, et al. The association between statin use and outcomes potentially attributable to an unhealthy lifestyle in older adults. Value Health. 2011;14(4):513-520.
Clinical Epidemiology

\section{Publish your work in this journal}

Clinical Epidemiology is an international, peer-reviewed, open access journal focusing on disease and drug epidemiology, identification of risk factors and screening procedures to develop optimal preventative initiatives and programs. Specific topics include: diagnosis, prognosis, treatment, screening, prevention, risk factor modification, systematic

\section{Dovepress}

reviews, risk \& safety of medical interventions, epidemiology \& biostatical methods, evaluation of guidelines, translational medicine, health policies \& economic evaluations. The manuscript management system is completely online and includes a very quick and fair peer-review system, which is all easy to use. 